Vol. 1, No 1, 2016

\title{
VANET EXPERIMENTAL RESULTS ON DETECTING TRAFFIC CONGESTION USING SINR AND DELAY
}

\author{
Ng Chee Yee ${ }^{1}$, Joanne Mun-Yee Lim ${ }^{1 *}$, Anis Fariza ${ }^{1}$ \\ ${ }^{1}$ Faculty of Engineering \& the Built Environment, SEGi University, Malaysia \\ *Email: joanne@segi.edu.my
}

\begin{abstract}
Vehicular ad-hoc network (VANET) is a wireless network that is formed by vehicles that are connected to each other through ad hoc formation. Since nodes in VANET are highly mobile moving in unpredictable environments, therefore VANET should be made adaptable. In order to have an adaptable VANET, several parameters such as end to end delay and Signal to Interference Noise Ratio (SINR), are used to detect traffic congestion/transmission signal degradation. In this paper, several experiments were conducted to test the suitability of SINR and end to end delay in detecting traffic congestion/transmission signal degradation in VANET. The experiments were conducted in congested, moderate and traffic free conditions in an urban area of Klang, Malaysia. Results showed that under different traffic congestions and transmission environments, SINR and end to end delay showed different readings, which proved the suitability of SINR and end to end delay in detecting traffic congestion and transmission degradation in VANET.
\end{abstract}

Keywords: SINR, VANET, End to end Delay, Congestion Detection, Experimental

\subsection{INTRODUCTION}

Vehicular ad-hoc network (VANET) is a self-organized network that is formed by connecting vehicles to improve driving safety and traffic management. There are a few types of communications provided in VANET. Firstly, vehicle to vehicle (V2V) is a wireless ad-hoc network where vehicles communicate with another vehicle without the support of infrastructure. On the other hand, vehicle to infrastructure (V2I) communication involves communication between vehicles and infrastructures. Both V2V and V2I are important to ensure safety in Intelligence Transport Systems (ITS).

In VANET, nodes communicate with the use of DSRC standard that employs the IEEE 802.11p standard for wireless communication. VANET has wide network size due to highly mobile VANET nodes, move at random and unpredictable environments. Therefore, VANET should be made adaptable to ensure transmission performance under different transmission environments. In order to ensure adaptability in VANET, several parameters such as SINR and end to end delay are used to detect traffic congestion/transmission signal degradation. 
In this paper, the researchers have conducted several experiments to test SINR and end to end delay under different transmission environments namely; congestion, moderate and traffic free conditions. The main contributions of this work are as follows: Firstly, the proposal is for two parameters, SINR and end to end delay, to detect traffic congestion and transmission signal degradation. Secondly, an experiment was conducted to obtain SINR and end to end delay readings under different transmission environments. Experimental results showed that the SINR and end to end delay change according to transmission degradation quality, which make them suitable to be used in VANET as congestion/transmission environment detection, so that transmissions in VANET can be adapted according to different transmission environments.

The remaining part of the paper is organized as follows. Section 2 discusses the related work. Section 3 explains about the methodology used to measure traffic condition. Section 4 presents the results and analysis of the research work. Section 5 concludes the paper.

\subsection{RELATED WORK}

VANET is an emergent technology that is used to create mobile network with the use of moving cars as nodes in a network. However, there are limited parameters to detect traffic congestion/transmission degradation.

The proposed algorithm in (Bazzi, Zanella, and Masini 2015) enables each vehicle in the network to detect and quantify the level of traffic congestion in a completely distributed way, independent of any supporting infrastructure by keeping vehicle's overall knowledge about congestion to be consistent, despite of short-term changes in vehicle's motion. However, it does not provide any information about intensity or volume of the traffic on road. In (Lin and Deng 2015), GPS is used to find nodes position under congestion. However, GPS auto arranges the navigation path without considering the traffic data. Studies in (Cheng, Ling, and Sadler 2015) implemented a particle swarm optimization (PSO) in which a centre particle is adopted for increasing the convergence speed. The advantage of this proposed idea is its fixed roadside units (RSUs), and fixed wireless sensor nodes, in which RSUs and sensors are deployed along the road acting as access points (APs) so that sensors may persistently detect road conditions and report them. Somehow, it is not suitable for non-uniform vehicle population as it increases the difficulty of communication in this network. In (C. Shi, Berry, and Honig 2014) safety-message transmission in VANET causes congestion under emergency communication priority. Hence, by introducing cognitive radio (CR) enabled VANET (CR VANET), where CR devices are able to detect possible idle spectrum for VANET communications and assist timely broadcast safety-message. Proposed algorithm has larger packet reception probability (PRP) and lower transmission delay as compared with conventional VANET broadcasting. However, fair channel allocation schemes among vehicles cause mutual interference.

In (Shrivastava and Agrawal 2014), combinations of probabilistic techniques, clustering approach and incorporating interference as noise in DS-CDMA method without using interference cancelation techniques are used to attain precise and approximation estimation of SINR distribution in wireless sensor networks (WSN). This calculates WSN's throughput and capacity, which is an important parameter for performance measuring in such networks. Unfortunately, there is an effect of path-loss as one channel parameter on the 
SINR distribution, for both sensors layer and headers layer. By assessing network connectivity through conductance of network measurement and finding bottleneck location of the network, a relay node is used to improve the connectivity at the bottleneck(F. Shi et al. 2015). This helps to minimize the ratio of the boundary link connectivity coefficients to the number of nodes in a set. However, there are network connectivity problems from data communication point of view. In (Haraty, Mohammadi, and Mehbodnia 2014), an adapting transmits beam formers and linear receiver filters in a Time Division Duplex Multiple-Input Multiple-Output (MIMO) interference network version of MAX-SINR algorithm is proposed. Bi-directional training in the forward direction is used to measure received SINR and interference prices. However, the large system analysis may not be possible to provide insight of the channel. Channel quantization (Hadded et al. 2015) for block diagonalization with limited feedback is used to approach the maximum capacity gain of multiple-input multiple- output (MIMO) broadcast channel (BC). An efficient channel state information (CSI) quantization technique for block diagonalization (BD) which is preceded by systems with limited feedback is proposed where the users are supported by a base station that is selected by dynamic scheduling. Advantage of this proposed study is to support multiple users at the same time and frequency resource by pre-cancelling inter-user interference using a simple inverse or inverse-like operation. However, when channel state information (CSI) knowledge is limited at the transmitter, the performance of the BD may be degraded because inter-user interference cannot be completely eliminated.

In order to measure time delay, (Lim et al. 2016) (Milojevic and Rakocevic 2014) ultra-short pulse using autocorrelation methodology was used. This is helpful in achieving high accuracy and precise synchronization. However, based on studies(Milojevic and Rakocevic 2014), capturing time of sliding correlation method is too long to be used in telephone time service. Besides, estimation of signal parameters via rotation invariance techniques (ESPRIT) with extended improved spatial smoothing techniques were used (Sun et al. 2016) to measure time delay for radar signals with higher accuracy. This method has a constant bias that is small enough to be compared with the accuracy requirement.

Based on current literature review thus far, there are a few methods that can be used to adapt MAC to VANET. MAC protocols in ad-hoc networks can be classified according to how channels are shared. Under heavy traffic conditions, carrier sense multiple accesses with collision avoidance (CSMA/CA) suffer from a high collision probability, particularly in the presence of hidden terminals. Furthermore, the adoption of the request-to-send/clear-to-send (RTS/CTS) mechanism is not effective when a high data rate is required. Therefore, orthogonal frequency-division multiple-access (OFDMA) based MAC protocol is introduced. However, there is less resource allocation in the frequency domain. Therefore, in (Moon et al. 2014) and (Jin, Hua, and Cao 2014), to ensure that all the vehicles have enough time to send safety messages without collisions and to reduce the end-to-end delay, a study has been conducted to achieve less transmission delay for safety application. Fairness, packet loss and stability are 
critical performance metrics for complex applications such as multimedia applications such as video or audio. Therefore, a study (Huang et al. 2016) is used to adapt the transmission probability at the MAC layer to reduce interference and maximise the singlehop throughput between adjacent nodes. This study shows that vehicular density can change rapidly creating heterogeneous scenarios.

Based on the aforementioned literature reviews, adaptability in VANET is important to ensure efficient packet transmission. Therefore, selecting parameters to detect transmission suitability is important to ensure adaptability and efficient packet transmission. However, the current research work lacks in experimental research in verifying the suitability of parameters in detecting change of environment in VANET. In this paper, SINR and end to end delay are tested in experimental setups to verify their suitability and efficiency in detecting change of VANET transmission environment.

\subsection{METHODOLOGY}

In order to detect a change of VANET environment, SINR and end to end delay are used as parameters to inform VANET of the change or deteriorating performance transmission environment, so that necessary measures can be taken to increase thr transmission performance by ensuring adaptability.

SINR measures signal strength with background noise. This allows SINR to be used to represent VANET packet transmission. High interference results in low SINR, indicates that the traffic condition in a certain area is congested. Therefore, by observing results of SINR, the traffic congestion state can be determined.

On the other hand, time delay is also one of the parameters proposed to detect changes of VANET transmission environment. If a packet takes longer time to reach its users, it represents that the specific area is congested. On the other hand, if shorter time is taken to send a similar size packet, this represents a traffic free VANET transmission environment. Hence, by observing results of time delay, the traffic condition can be determined.

\subsection{THE PROPOSED SINR AS VANET PARAMETER TO DETECT CHANGES OF VANET ENVIRONMENT}

Under congested state, mobile phones and its network commonly fail to function in stadiums, concerts or any other crowded festivals. This is because of an increase of spectrum congestion for mobile-phone use in a certain area. Therefore, if that area is too crowded, the spectrum is re-used over and over again by multiple cell sites which uses the same frequency. Hence, no phone calls or messages could be reached out from that specific crowded area. 
Theoretically, SINR will be higher in congested traffic compared to moderate traffic and traffic free. Therefore, SINR can be used to act as parameter of VANET to determine the traffic congestion.

Due to a large scale of VANET network, and the similarity between SINR in VANET transmission environment, and mobile phones network in a congested state, an experiment is conducted by using 9 phones to represent 9 cars in a congested traffic, 6 phones to represent 6 cars in a moderate traffic and 3 phones to represent 3 cars in a traffic free VANET transmission environment as shown in Figure 1. With the aid of an android app named as Net monitor, a phone is used to determine the SINR.

To detect traffic congestion, the equation of SINR is shown in equation (1). The higher the SINR represents congested traffic and vice versa.

$$
\mathrm{SINR}=\frac{a}{b+e}
$$

where $\mathrm{a}=$ Signal strength

$\mathrm{b}=$ Environmental disturbance (noise)

$\mathrm{e}=$ Environmental disturbance (interference)

1(a)

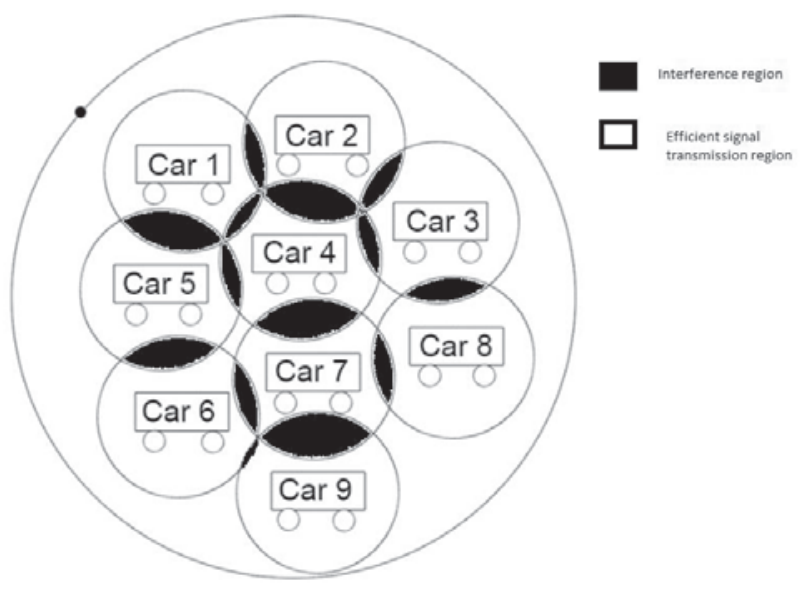

Figure 1. (a): Congested traffic.

1 (b) 


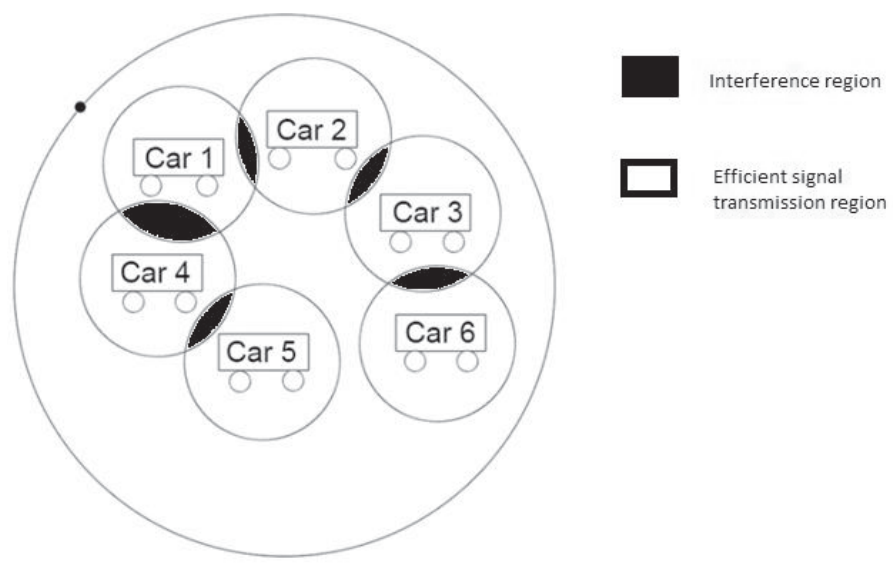

Figure 1. (b): Moderate traffic

1(c)

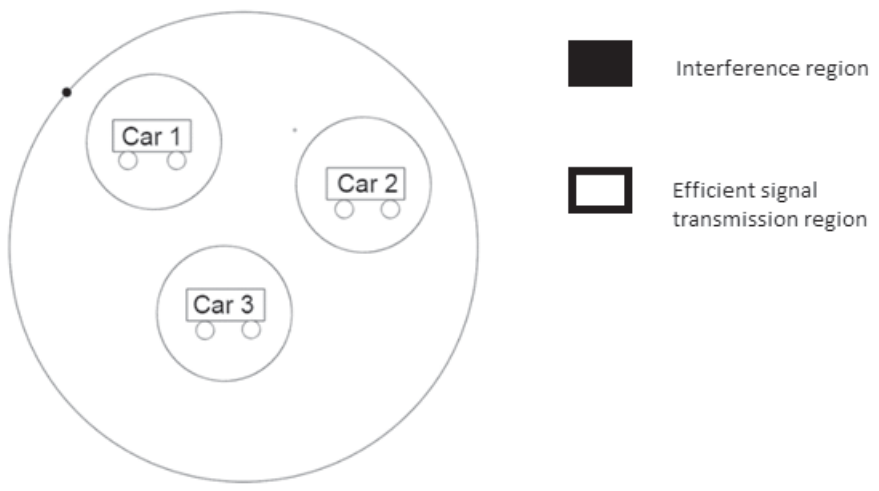

Figure 1. (c): Traffic free. 


\subsection{THE PROPOSED END TO END DELAY AS VANET PARAMETER TO DETECT CHANGES OF VANET ENVIRONMENT}

End to end delay is one of the proposed methods to measure changes of transmission environment in VANET. If the delay is shorter, it represents traffic free transmission environment. If the delay is higher, it represents traffic congestion transmission environment.

Due to the large scale of VANET system, in this experiment, a $1.5 \mathrm{mb}$ JPEG format is transmitted from one phone to another phone with several Bluetooth version 4.0 wireless connections turned on from phones to create interferences, hence replicating the VANET transmission environment. Time taken for files to be transferred completely are recorded down for experimental purpose. For congested traffic, 9 Bluetooth wireless networks from phones are turned on. While for moderate traffic, 6 Bluetooth wireless networks are turned on. Lastly for traffic free transmission condition, 3 Bluetooth wireless networks are turned on.

Bluetooth has limit wireless network coverage up to 10 meters. So, it is suitable to be used in between vehicles and nearby roadside units. Connected devices will be set in a specific frequency to allow packet to be able to send through. Ideally, the time taken for data to send through will be interfered by other wireless devices or networks due to the same usage of $2.4 \mathrm{GHz}$ radio frequency band. Bluetooth is a short range wireless interconnection gadget that is used in cellular phones, computers, tablets and etc. based on frequency-hopping spread spectrum (FHSS) radio technology. However, Bluetooth devices use $2.4 \mathrm{GHz}$ radio frequency band which is a similar band with Wi-Fi, telephone, car alarm, microwave oven and video devices. This means that the other wireless devices or networks can interfere with Bluetooth data transfer rate.

Based on the proposed experimental studies, by calculating the time delay from Bluetooth data transfer rate, the VANET transmission condition can be represented. The amount of delay represents the traffic conditions in VANET. Due to the large scale of VANET, the above method is used to conduct this experiment.

\subsection{RESULT AND ANALYSIS}

The performance of proposed experiments is evaluated with the use of Net monitor applications, Bluetooth v4.0, and open Google map application. These experiments are conducted in Glenmarie Cove, Klang as shown in Figure 2. Parameters used for this experiment are shown in Table 1 and 2.

Table 1: SINR Experiment Parameters

\begin{tabular}{|l|l|}
\hline Parameter & Value \\
\hline Environment Signal & $-85 \mathrm{dBm}$ \\
\hline $\begin{array}{l}\text { Number of phones used to represent traffic } \\
\text { free }\end{array}$ & 3 \\
\hline $\begin{array}{l}\text { Number of phones used to represent } \\
\text { moderate traffic }\end{array}$ & 6 \\
\hline
\end{tabular}




\begin{tabular}{|l|l|l|}
\hline $\begin{array}{l}\text { Number of phones used to represent } \\
\text { congested traffic }\end{array}$ & 9 \\
\hline
\end{tabular}

Table 2: Delay Experiment Parameters

\begin{tabular}{|l|l|}
\hline Parameters & Value \\
\hline Bluetooth version & 4.0 \\
\hline Size of packet & $1.4 \mathrm{MB}$ \\
\hline Type of packet & JPEG format image \\
\hline Transmission Range & $<10$ meter \\
\hline $\begin{array}{l}\text { Number of Bluetooth used to represent } \\
\text { traffic free }\end{array}$ & 3 \\
\hline $\begin{array}{l}\text { Number of Bluetooth used to represent } \\
\text { moderate traffic }\end{array}$ & 6 \\
\hline $\begin{array}{l}\text { Number of Bluetooth used to represent } \\
\text { congested traffic }\end{array}$ & 9 \\
\hline
\end{tabular}

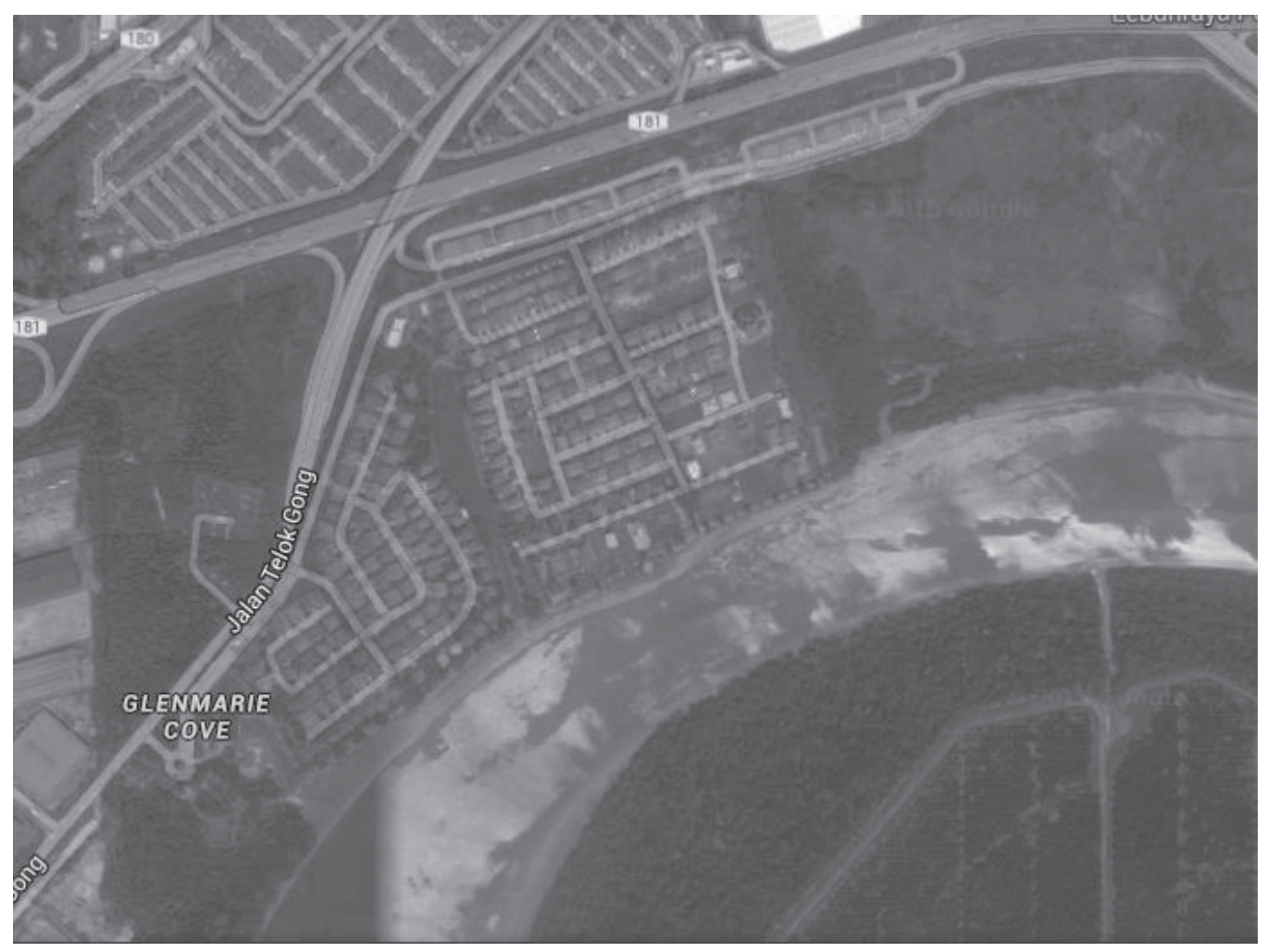

Figure 2. Glenmarie Cove, Klang. 
Table 3 shows the readings of SINR obtained from congested traffic, moderate traffic and traffic free VANET transmission environments. Table 4 shows the reading of time delay from congested traffic, moderate traffic and traffic free in seconds.

Table 3. SINR values for different traffic conditions

\begin{tabular}{|c|c|c|c|c|}
\hline \multirow{2}{*}{$\begin{array}{l}\text { Traffic } \\
\text { type }\end{array}$} & $1^{\text {st }}$ test & $2^{\text {nd }}$ test & $3^{\text {rd }}$ test & \multirow{2}{*}{$\begin{array}{l}\text { Average } \\
\text { SINR }\end{array}$} \\
\hline & \multicolumn{3}{|c|}{$S I N R=\frac{\text { signal strength }}{\text { noise }+ \text { interference }}$} & \\
\hline $\begin{array}{l}\text { Congested } \\
\text { traffic } \\
(9 \text { nodes })\end{array}$ & $\begin{array}{l}\frac{(-87)}{(-87)+(-85)} \\
=0.5174\end{array}$ & $\begin{array}{l}\frac{(-83)}{(-80)+(-85)} \\
=0.5030\end{array}$ & $\begin{array}{l}\frac{(-85)}{(-71)+(-85)} \\
=0.5449\end{array}$ & 0.5219 \\
\hline $\begin{array}{l}\text { Moderate } \\
\text { traffic } \\
(6 \text { nodes })\end{array}$ & $\begin{array}{l}\frac{(-87)}{(-89)+(-85)} \\
=0.5\end{array}$ & $\begin{array}{l}\frac{(-83)}{(-81)+(-85)} \\
=0.5\end{array}$ & $\begin{array}{l}\frac{(-85)}{(-81)+(-85)} \\
=0.512\end{array}$ & 0.5040 \\
\hline $\begin{array}{l}\text { Traffic } \\
\text { free } \\
(3 \text { nodes })\end{array}$ & $\begin{array}{l}\frac{(-87)}{(-93)+(-85)} \\
=0.4888\end{array}$ & $\begin{array}{l}\frac{(-83)}{(-90)+(-85)} \\
=0.4743\end{array}$ & $\begin{array}{l}\frac{(-85)}{(-87)+(-85)} \\
=0.4942\end{array}$ & 0.4858 \\
\hline
\end{tabular}

In Table 3 above, SINR is observed to change according to different traffic conditions. SINR is the highest when the number of nodes is the highest, which represents congested traffic. SINR is moderately low under moderate nodes transmission condition. Lastly, SINR is shown to be the lowest under traffic free condition, lowest number of nodes. The above experiment results show that SINR vary according to traffic conditions and number of nodes. Therefore, SINR is shown to be a reliable value to represent VANET transmission condition.

Table 4: Time delay for different traffic conditions

\begin{tabular}{|l|c|c|c|c|}
\hline Traffic type & $\begin{array}{l}\mathbf{1}^{\text {st }} \\
(\text { delay) }\end{array}$ & $\mathbf{2}^{\text {nd }}$ test (delay) & $\mathbf{3}^{\text {rd }}$ test (delay) & Average delay \\
\hline $\begin{array}{l}\text { Congested traffic } \\
(9 \text { nodes) }\end{array}$ & $11.13 \mathrm{~s}$ & $10.97 \mathrm{~s}$ & $11.06 \mathrm{~s}$ & $11.05 \mathrm{~s}$ \\
\hline $\begin{array}{l}\text { Moderate traffic } \\
(6 \text { nodes })\end{array}$ & $9.91 \mathrm{~s}$ & $9.86 \mathrm{~s}$ & $9.84 \mathrm{~s}$ & $9.87 \mathrm{~s}$ \\
\hline
\end{tabular}




\begin{tabular}{|l|l|l|l|l|}
\hline & & & & \\
\hline $\begin{array}{l}\text { Traffic free } \\
(3 \text { nodes })\end{array}$ & $8.75 \mathrm{~s}$ & $9.23 \mathrm{~s}$ & $9.25 \mathrm{~s}$ & $9.08 \mathrm{~s}$ \\
\hline
\end{tabular}

Different time delay represents different VANET transmission environment. In Table 4, time delay is the highest where 9 nodes are placed in a network to represent congested network. On the other hand, 6 nodes are used to represent moderate traffic, where a moderately lower delay is observed in Table 4. Lastly, traffic free traffic is represented using 3 nodes, where lowest delay is observed. Experimental results show the efficiency of delay in representing different traffic conditions.

\subsection{CONCLUSION}

VANET is an emerging technology that is soon to be deployed in every car network. Car network should be made more adaptable to various environments and nature of VANET. Therefore, an accurate and efficient parameter to detect traffic congestion in VANET is important. In this work, two parameters to detect traffic condition and change of VANET transmission environment, SINR and end to end delay, are proposed. Since vehicles in VANET are highly mobile, real-time detection is focused to be the main requirement of the parameters. In these experiments, SINR and end to end delay are measured under different transmission environments. SINR and end to end delay are shown to be different under different traffic conditions. Results show that parameters introduced are able to achieve realtime detection in VANET. With these parameters, VANET can be made adaptable to suit changing transmission environments.

\subsection{ACKNOWLEDGEMENT}

This research is funded by SEGi University.

\subsection{REFERENCES}

- Bazzi, Alessandro, Alberto Zanella, and Barbara Mavi Masini. 2015. "An OFDMABased MAC Protocol for Next-Generation VANETs.” IEEE Transactions on Vehicular Technology 64(9): 4088-4100.

- Cheng, Maggie X., Yi Ling, and Brian M. Sadler. 2015. "SINR-Based Connectivity Enhancement in Wireless Ad Hoc Networks." IEEE International Conference on Communications 2015-September: 3696-3701.

- Hadded, Mohamed et al. 2015. "TDMA-Based MAC Protocols for Vehicular Ad Hoc Networks: A Survey, Qualitative Analysis, and Open Research Issues." IEEE Communication Surveys \& Tutorials 17(4): 2461-92.

- Haraty, Mahdi, Abbas Mohammadi, and Mohammad Mehbodnia. 2014. "Estimation of SINR Distribution Function in Clustered Wireless Sensor Networks Using Statistical Distribution Modeling." In Telecommunications (IST), 2014 7th International Symposium on, IEEE, 740-45.

- Huang, X.-L. et al. 2016. "Historical Spectrum Sensing Data Mining for Cognitive 
Radio Enabled Vehicular Ad-Hoc Networks." IEEE Transactions on Dependable and Secure Computing 13(1): 59-70. https:/www.scopus.com/inward/record.uri?eid=2-s2.084962609258\&partnerID=40\&md5=62963a22d88ca049f1746013e6345e08.

- Jin, Xiaozhen, Yu Hua, and Yuanhong Cao. 2014. "Research on A New Method of Time Delay Measurement in Telephone Time Service." In IEEE International Frequency Control Symposium (FCS), IEEE, 1-3.

- Lim, Joanne Mun Yee, Yoong Choon Chang, Mohamad Yusoff Alias, and Jonathan Loo. 2016. "Joint Optimization and Threshold Structure Dynamic Programming with Enhanced Priority Scheme for Adaptive VANET MAC." Wireless Networks 22(3): 897913. "http://dx.doi.org/10.1007/s11276-015-1009-x.

- Lin, Chun Cheng, and Der Jiunn Deng. 2015. "Optimal Two-Lane Placement for Hybrid VANET-Sensor Networks.” IEEE Transactions on Industrial Electronics 62(12): 788391.

- Milojevic, M, and V Rakocevic. 2014. "Distributed Road Traffic Congestion Quantification Using Cooperative VANETs." Ad Hoc Networking Workshop (MEDHOC-NET), 2014 13th Annual Mediterranean: 203-10.

- Moon, Sung Hyun, Sang Rim Lee, Jin Sung Kim, and Inkyu Lee. 2014. "Channel Quantization for Block Diagonalization with Limited Feedback in Multiuser MIMO Downlink Channels." Journal of Communications and Networks 16(1): 1-9.

- Shi, Changxin, Randall A. Berry, and Michael L. Honig. 2014. "Bi-Directional Training for Adaptive Beamforming and Power Control in Interference Networks." IEEE Transactions on Signal Processing 62(3): 607-18.

- Shi, Fan et al. 2015. "High Precise Time-Synchronization Based on Ultrashort Pulse." In Joint Conference of the IEEE International Frequency Control Symposium \& the European Frequency and Time Forum, IEEE, 290-92.

- Shrivastava, Disha, and Arun Agrawal. 2014. "Traffic Congestion Detection in Vehicular Adhoc Networks Using GPS." In IEEE International Conference on Computational Intelligence and Computing Research, IEEE, 1-7.

- $\quad$ Sun, Meng, Cedric Le Bastard, Yide Wang, and Nicolas Pinel. 2016. "Time-Delay Estimation Using ESPRIT With Extended Improved Spatial Smoothing Techniques for Radar Signals.” IEEE Geoscience and Remote Sensing Letters 13(1): 73-77. http://ieeexplore.ieee.org/document/7335605/. 